\title{
Organic-Inorganic Particles as Labels to Trace Biological Signaling Pathways
}

\author{
A.L. Koh ${ }^{1,2}$ and R. Sinclair ${ }^{1}$ \\ ${ }^{1}$ Department of Materials Science and Engineering, Stanford University, Stanford CA 94305, USA \\ ${ }^{2}$ Currently at: Department of Materials, Imperial College London, London SW72AZ, UK
}

Communication in cells takes place in the form of cell signaling. The latter governs basic cellular activities and coordinates cell actions. Errors in cellular information processing are responsible for diseases such as cancer. Thus, by understanding cell signaling, diseases may be treated effectively. Many intracellular signaling pathways lead from cell-surface receptors to the nucleus, where they alter gene transcription [1]. The JAK-STAT [2] signaling pathway provides one of the most direct routes and is therefore commonly investigated.

Typical methods to study intracellular signaling events include mass spectrometry and flow cytometry. One innovative approach is to make use of labels (nanoparticles) which have been functionalized with appropriate antibodies that target STAT proteins, which is the objective of the present work. In this paper, we demonstrate the use of Composite Organic-Inorganic Nanoparticles (COINs) [3-5] as labels to trace the STAT6 signaling pathway of cells. COINs are novel surfaceenhanced Raman (SER) scattering nanoclusters formed by aggregating inorganic silver nanoparticles in the presence of Raman-active molecules (Fig. 2). They are then encapsulated with organic constituents bovine serum albumin and glutaraldehyde, functionalized with STAT6 antibodies, and then conjugated to U937 monocytic leukemia cell lines which have been treated with the cytokine Interleukin-4 (IL-4). Successful binding between COINs and cells is verified using SER spectroscopy (Fig. 2). Transmission electron microscopy (TEM) was performed on the COIN-cell conjugates. Samples were post-fixed, stained, embedded, ultramicrotomed and studied using a Philips CM20 FEG-TEM operated at 120kV. TEM analyses of the cell sections show that the STAT6-COINs entered the stimulated cells, traced the STAT6 signaling pathway and translocated to the cell nucleus (Fig. 3). Thus, the use of COINs as labels to trace biological signaling pathways is demonstrated for the first time.

\section{References}

[1] B. Alberts et al., Molecular Biology of the Cell (Fourth Edition), Garland Science, New York (2002)

[2] T. Kisseleva et al., Gene 285 (2002) 1

[3] X. Su et al. Nano Lett. 5(1) (2005), 49-54.

[4] L. Sun et al. Nano Lett. 7(2) (2007), 351-356.

[5] A.L. Koh et al. Ultramicroscopy 109 (2008), 110-121.

[6] This research is supported by the Center for Cancer Nanotechnology Excellence Focused on Therapy Response (CCNE-TR) grant (NIH U54) and the Nanyang Technological University (Singapore) Overseas Scholarship. The authors would like to thank Drs. C.M. Shachaf and G.P. Nolan from the Department of Microbiology and Immunology at Stanford University for synthesizing and providing the samples, and for discussions. 

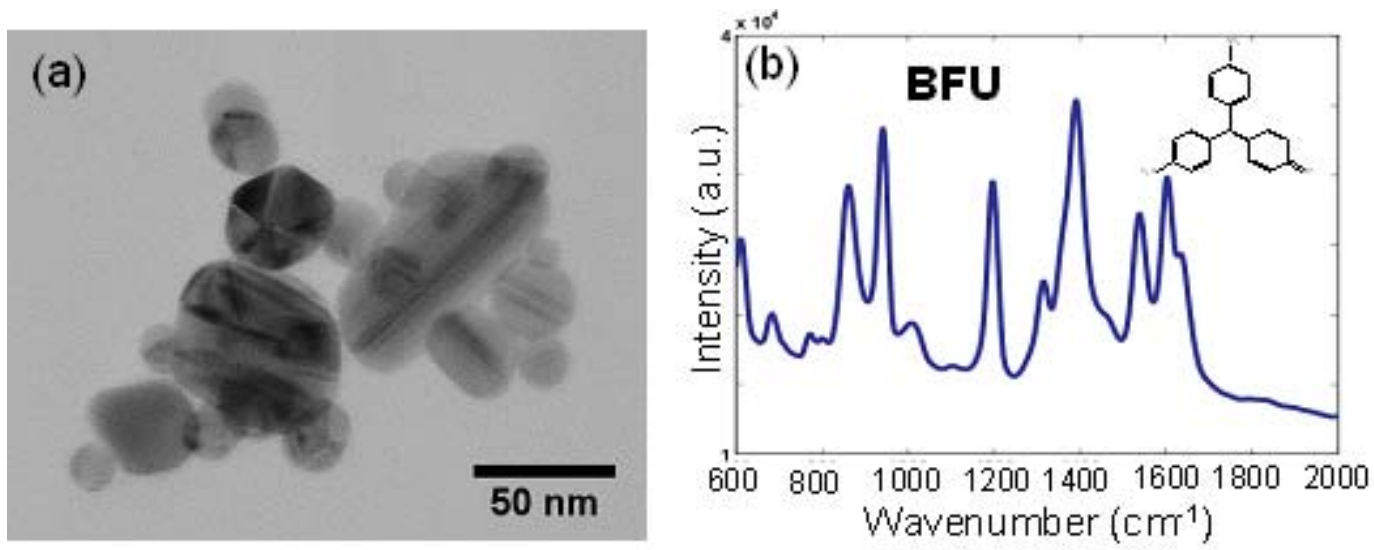

FIG 1. (a) TEM bright-field image of as-synthesized COINs and (b) its corresponding SER spectra.

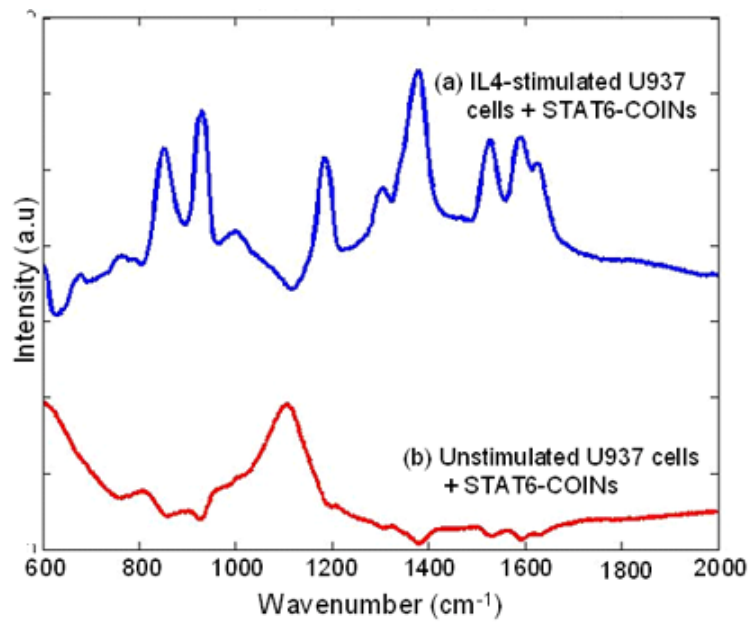

FIG 2. SER spectra of (a) IL4-stimulated U937 cells conjugated with STAT6-COINs and (b) the control sample of unstimulated U937 cells conjugated with STAT6-COINs, suggesting binding between cells and COINs in the former.
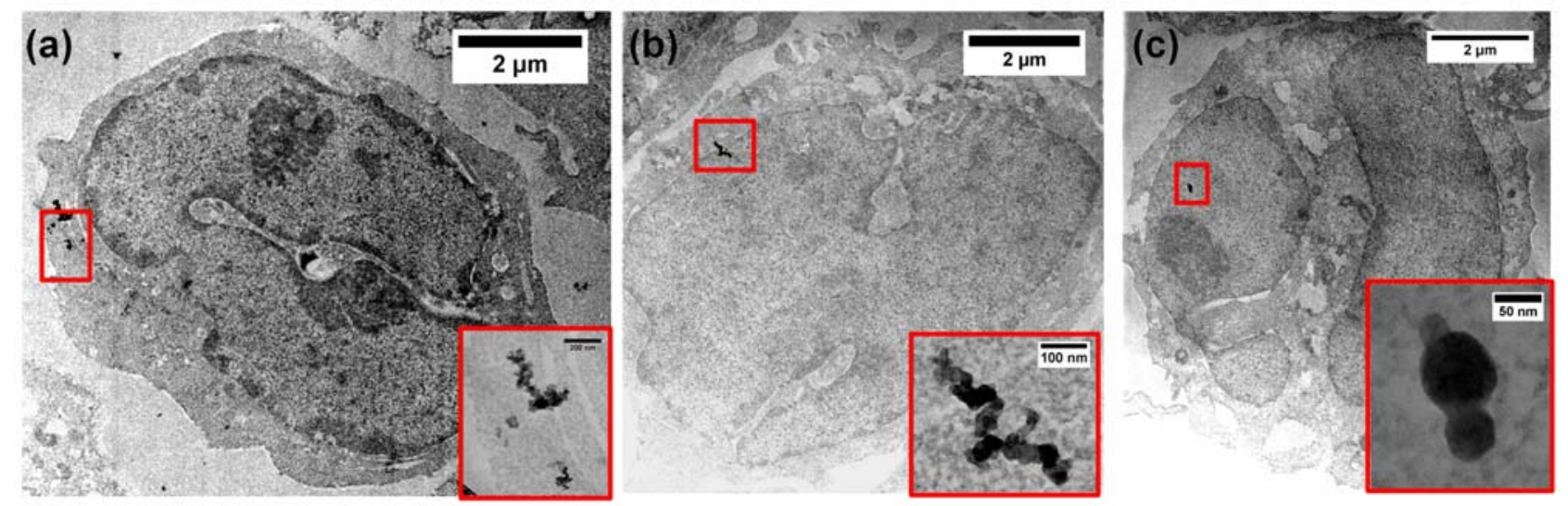

FIG 3. TEM bright-field images of COINs in the (a) cell membrane, (b) nuclear membrane and (c) nucleus, demonstrating their use as labels to trace the STAT6 signaling pathways. Higher magnification images of COINs inset. 